\title{
Quetiapine for the Treatment of Delirium
}

\author{
Stefanie B. Hawkins, PharmD1, Mason Bucklin, PharmD², Andrew J. Muzyk, PharmD*
}

${ }^{1}$ Emergency Medicine, University of Kansas Hospital, Kansas City, Kansas; '2Department of Pharmacy, University of Rochester Medical Center, Rochester, New York; ${ }^{3}$ Department of Pharmacy Practice, Campbell University School of Pharmacy and Health Sciences, Buies Creek, North Carolina, and Department of Psychiatry and Behavioral Sciences, Duke University, Durham, North Carolina.

BACKGROUND: Delirium is associated with high rates of morbidity and mortality in hospitalized medically ill patients. Haloperidol has historically been the agent of choice for the treatment of delirium, but recent studies have explored the efficacy of second-generation antipsychotics such as quetiapine. The unique pharmacology of quetiapine may allow it to treat delirium and provide sedation without causing significant extrapyramidal side effects.

PURPOSE: To evaluate the efficacy of quetiapine for the treatment of delirium.

DATA SOURCES: A search was conducted in MEDLINE and Embase (January 1960-December 2012) using keywords "quetiapine," "second-generation antipsychotic," "atypical antipsychotic," "delirium," and "agitation."

STUDY SELECTION AND DATA EXTRACTION: The search was limited to English-language articles and trials with treatment of delirium as the primary end point. Eight trials met this inclusion criterion.
DATA SYNTHESIS: Two randomized controlled trials, 5 open-label studies, and 1 retrospective cohort study evaluating quetiapine for the treatment of delirium were reviewed. One randomized controlled trial showed no differences in total mean delirium scores, but found the rate of delirium improvement was significantly shorter with quetiapine. The second randomized controlled trial showed the time to first resolution of delirium was shorter with quetiapine compared to placebo. Results of the open-label and retrospective cohort trials have also shown significant resolution of delirium from baseline and equal efficacy with quetiapine compared to amisulpride and haloperidol.

CONCLUSIONS: Quetiapine appears to be an effective and safe agent for the treatment of delirium in both general medicine and intensive care unit patients. The trials summarized suggest that quetiapine resolves symptoms of delirium more quickly than placebo and has equal efficacy compared to haloperidol and the atypical antipsychotic amisulpride. Further study is needed. Journal of Hospital Medicine 2013;8:215-220. (c) 2013 Society of Hospital Medicine
Delirium is an acute fluctuation in mental status that includes symptoms of inattention, disorganized thinking, and altered level of consciousness occurring over a short time period. ${ }^{1}$ Prevalence of delirium ranges from $10 \%$ to $30 \%$ in the hospitalized medically ill and ranges from $40 \%$ to $60 \%$ in intensive care unit (ICU) patients who are not receiving mechanical ventilation. 2,3 Patients experience delirium more often if they are of older age; have dementia, cancer, or acquired immune deficiency syndrome; have undergone surgery; are terminally ill; or have received multiple psychoactive medications, particularly benzodiazepines or opioids. ${ }^{3-7}$ Delirium is associated with high rates of morbidity and mortality, ${ }^{8}$ with patients more likely to develop complications such as pneumonia, decubitus ulcers, and long-term cognitive deficits. ${ }^{9-12}$ These complications, in turn, lead to longer hospital stays and increased costs of care. ${ }^{13,14}$

*Address for correspondence and reprint requests: Andrew J. Muzyk, PharmD, P.O. Box 3089-Pharmacy, Durham, NC 27710; Telephone: 919681-3438; Fax: 919-681-2741; E-mail: andrew.muzyk@duke.edu

Additional Supporting Information may be found in the online version of this article.

Received: October 24, 2012; Revised: January 8, 2013; Accepted: January 15, 2013

2013 Society of Hospital Medicine DOI 10.1002/jhm.2019

Published online in Wiley Online Library (Wileyonlinelibrary.com).
Currently, there are no antipsychotics approved by the US Food and Drug Administration for the treatment of delirium.

Both the 2002 American Society of Critical Care Medicine $^{12}$ and the 2004 American Psychiatric Association guidelines ${ }^{15}$ on delirium recommend haloperidol as the antipsychotic of choice due to its potent tranquilizing effect, lack of active metabolites, and limited anticholinergic and sedating side effects. However, when given intravenously or at high cumulative doses (generally $>35 \mathrm{mg} /$ day), haloperidol has been shown to cause QT interval prolongation potentially leading to torsades de pointes and sudden cardiac death. ${ }^{15}$ Recent research in delirium treatment has focused on the second-generation antipsychotics, and these studies have reported positive findings, ${ }^{16-29}$ although no significant differences have been found compared to haloperidol. ${ }^{16-18,22-24}$ Systematic reviews have also failed to show a significant difference in efficacy or safety between second-generation and first-generation antipsychotics and unfortunately report a number of limitations including poor study designs, small sample sizes, lack of a placebo control group, exclusion of ICU patients, and weak primary outcomes. ${ }^{8,30-32}$ Attempting to correct for a number of these limitations, recent research with quetiapine has reported promising results in 2 controlled studies. ${ }^{19,20}$ 
Quetiapine is a second-generation antipsychotic with a very low affinity for dopamine receptors and a very high affinity for serotonin receptors. ${ }^{33,34}$ Additionally, quetiapine has a high affinity for histamine and $\alpha 1$-adrenergic receptors, but a very low affinity for M1 muscarinic receptors. ${ }^{34}$ This mechanism of action may allow quetiapine to effectively treat delirium and provide sedation without causing significant extrapyramidal side effects associated with potent dopamine receptor antagonism or precipitating delirium through muscarinic receptor antagonism. Quetiapine has a rapid absorption and a short half-life (3-6 hours), giving it a quick onset of action and a fast elimination from the body. ${ }^{35}$ Unlike haloperidol, it is only available in oral dosage forms, but can be crushed to administer via enteral tube. Common reported adverse effects include somnolence, hypotension, and dizziness. Although quetiapine has been shown to prolong QTc, Harrigan et al. reported a mean increase in QTc from baseline of $5.7 \mathrm{msec}$; there was no significant effect on this change in the presence of a metabolic inhibitor, ketoconazole. ${ }^{36}$ The mean change in QTc from baseline with quetiapine was lower than the change reported with oral haloperidol, $5.7 \mathrm{msec}$ compared to $7.1 \mathrm{msec}$, respectively. No statistical comparison was performed between these 2 drugs on this measure. Quetiapine does carry a black box warning for increased mortality in elderly patients with dementia-related psychosis. ${ }^{35}$ However, this risk has also been found with first-generation antipsychotics. ${ }^{37-39}$ A recent study found this risk of sudden cardiac death extended to adult users of both firstand second-generation antipsychotics. ${ }^{40}$ In contrast, Elie et al. found no increased risk of mortality in elderly patients with delirium receiving antipsychoticsover $90 \%$ were prescribed either haloperidol or risperidone-in their nested case-controlled study. ${ }^{41}$ Quetiapine has demonstrated some benefit with limited side effects in studies utilizing antipsychotics for the treatment of delirium. The purpose of this review was to evaluate the role of quetiapine for the treatment of delirium.

\section{LITERATURE REVIEW}

We performed an English-language literature search of MEDLINE and Embase databases to identify journal articles published between January 1960 and December 2012. Keywords included "quetiapine," "secondgeneration antipsychotic," "atypical antipsychotic," "delirium," and "agitation." The search was limited to English-language articles and adult subjects $(>18$ years). Based on our review of abstracts, we included both controlled and noncontrolled trials as long as treatment of delirium was the primary focus. We eliminated case reports, foreign language articles, and poster presentations. We identified 8 trials ${ }^{19,20,24-29}$ that included 2 double-blind, randomized, placebocontrolled trials, which are described in the text below. ${ }^{19,20}$ Six other trials, 5 open-label ${ }^{25-29}$ and 1 retrospective cohort, ${ }^{24}$ are described in Table 1.

\section{Randomized Controlled Trials}

Tahir et al. published a double-blind, randomized, placebo-controlled trial examining the efficacy and tolerability of quetiapine in the treatment of delirium. ${ }^{19}$ Inclusion criteria were a Diagnostic and Statistical Manual of Mental Disorders-IV diagnosis of delirium and a Delirium Rating Scale Revised 98 (DRS-R-98) total score of at least 15 , indicating the presence of delirium. Subjects were excluded for major preexisting cognitive deficits, alcohol withdrawal, preexisting psychosis, substance dependence, inability to comply with the constraints of the trial, and concurrent medications that interact with quetiapine.

Forty-two general medicine subjects were enrolled and randomized in the study with no difference in baseline characteristics. Patients received either placebo or quetiapine $25 \mathrm{mg}$ once daily. Doses were titrated by $25 \mathrm{mg}$ daily to a maximum daily dose of $175 \mathrm{mg}$ in divided doses. The primary end point was DRS-R-98 total mean score assessed on days 1, 2, 3, 4,7 , and 10, with follow-up assessment on day 30 . Secondary outcome measures were Mini-Mental State Examination (MMSE), the Brief Psychiatric Rating Scale, and the Clinical Global Improvement Scale. Tolerability was assessed using the Abnormal Involuntary Movements Scale and by clinical examination.

No differences in total mean DRS-R-98 score at individual time points reached statistical significance, but these scores improved more quickly in the quetiapine group than placebo. The secondary outcome of rate of delirium improvement on severity score did reach statistical significance; differences on mean severity scores were $0.827 \pm 0.37(P=0.026)$, suggesting that severity scores improved $82 \%$ more quickly than placebo. There were no significant differences between groups for any of the other secondary outcomes. Seven patients died within 30 days of entering the study (4 in the quetiapine group and 3 in the placebo group) due to serious medical conditions and not due to study medication as determined through clinical review. One patient withdrew from quetiapine due to sedation. Results of this study are limited by a small sample size, as it was underpowered to detect a statistically significant difference in the primary outcome. Other limitations include subjects who were older, with mean age of 84 years, which may have prevented titration of quetiapine, and subjects with minor cognitive deficits were included, which may have reduced the impact of treatment on the study outcomes. Additionally, strict criteria for exclusion kept those patients most likely to develop delirium from being included, limiting the studies external validity. Finally, the use of DRS-R-98 mean total and 


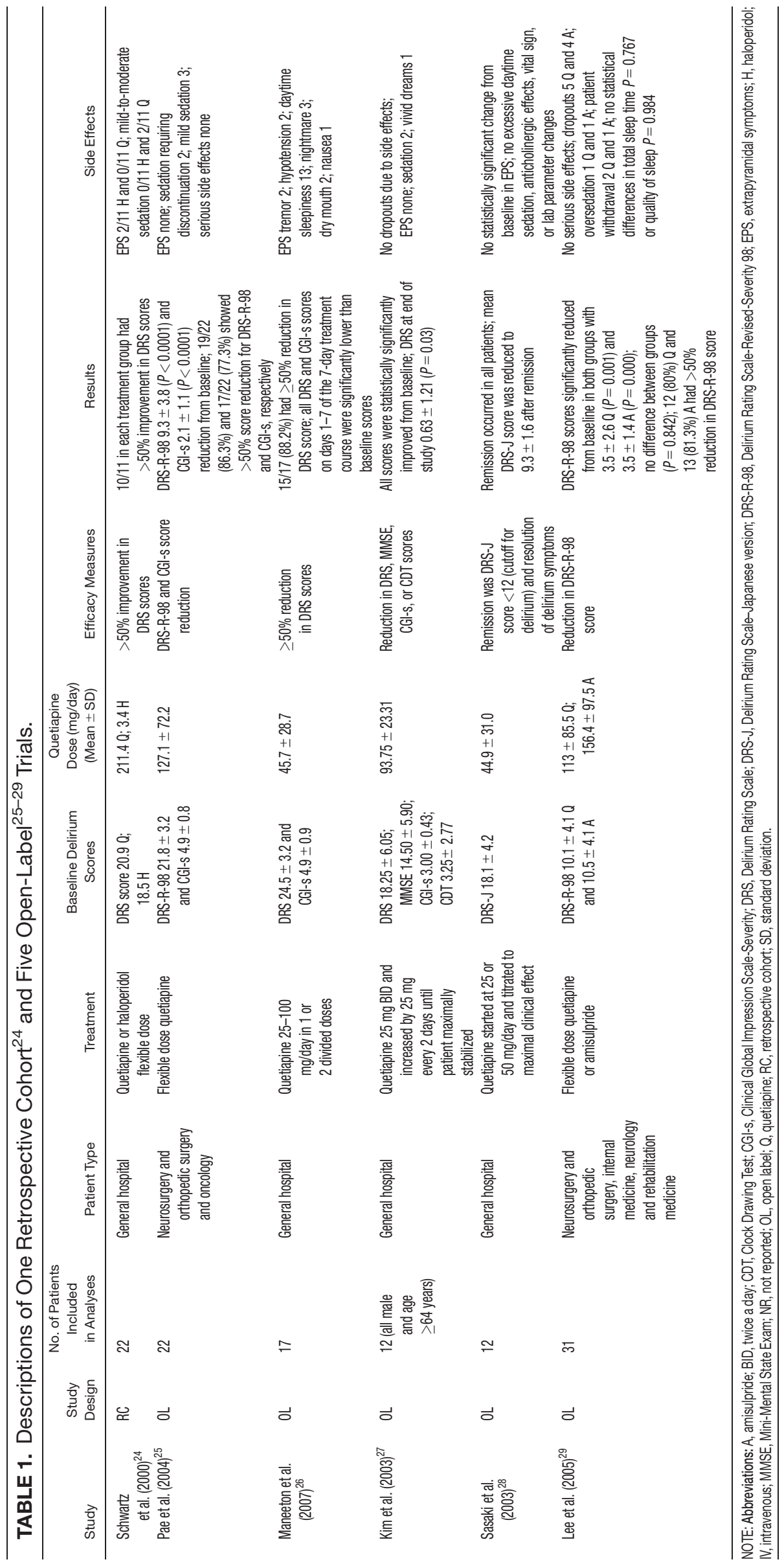


severity scores is subject to error as outliers in the mean could potentially skew the results.

Devlin et al. investigated the efficacy and safety of quetiapine for ICU delirium in critically ill patients in a double-blind, randomized, placebo-controlled trial. ${ }^{20}$ Inclusion criteria were an Intensive Care Delirium Screening Checklist Score (ICDSC) $>4$, which indicates the presence of delirium, an order for asneeded haloperidol, and tolerating enteral nutrition. Patients were excluded if they had a complicating neurologic condition, current treatment with dexmedetomidine or with medications that interact with quetiapine, baseline QTc interval $>500$, pregnancy, or poor prognosis. Thirty-six critically ill subjects were randomized with no significant differences in baseline characteristics including exposure to fentanyl, haloperidol, and benzodiazepines, and in particular, midazolam. If the subject received at least 1 dose of asneeded haloperidol in the previous 24 hours, then either placebo or quetiapine was given. Quetiapine was initiated at $50 \mathrm{mg}$ every 12 hours and titrated up by $50 \mathrm{mg}$ every 12 hours to a maximum dose of $200 \mathrm{mg}$ every 12 hours. Patients could receive intravenous haloperidol, 1 to $10 \mathrm{mg}$ up to every 2 hours as needed. The primary outcome was time to first resolution of delirium defined as time from administration of the first dose of study drug until an ICDSC $<3$ was first detected, indicating an absence of delirium. Secondary outcomes were total hours in delirium, total hours spent "deeply sedated" (Sedation-Agitation Scale $[\mathrm{SAS}]<2$ ) or agitated (SAS $>5$ ), episodes of subject-initiated device removal, use of haloperidol therapy including total dose in milligrams, number of doses and number of days of therapy, the use of sedatives (converted to midazolam equivalents) and analgesics, duration of study-drug administration, average daily and maximum study-drug dose, length of mechanical ventilation, duration of both ICU and hospital stay, hospital mortality, and disposition of subjects after hospital discharge. Safety measures were total number of adverse and serious adverse events, episodes of somnolence, incidence of extrapyramidal symptoms, and episodes of QTc interval prolongation.

The time to first resolution of delirium was shorter with quetiapine compared to placebo (median [interquartile range]: 1.0 [0.5-3.0] vs 4.5 days [2.0--7.0]; $P=0.001)$. Resolution of delirium occurred at least once in all quetiapine patients and in $78 \%$ of placebo patients $(P=0.05)$. Statistical significance with quetiapine was reached on the following secondary end points: time spent in delirium (36 [12-87] vs 120 hours [60-195], $P=0.006)$; shorter duration of study drug (102 [84-168] vs 186 hours [108-228], $P=0.04)$; lower daily study drug dose (110 [88-191] vs $210 \mathrm{mg}$ [116-293], $P=0.01)$; less upregulation of the study medication dose (200 [100-313] vs $375 \mathrm{mg}$ [25-400], $P=0.02)$; fewer hours of agitation (6 [038 ] vs 36 hours [11-66], $P=0.02)$; shorter duration of haloperidol therapy (3 [2-4] vs 4 days [3-8], $P=0.05)$; and fewer days of fentanyl $(0[0-3]$ vs 4 days [1-9], $P=0.03$ ). There were no significant differences between groups for any other secondary outcomes. As-needed haloperidol use in the quetiapine versus the placebo group was lower but not statistically significant ( 1.9 vs $4.3 \mathrm{mg}$ per day; $P=0.26$ ). Two main limitations were a small sample size and strict exclusion criteria. Additionally, the primary end point of time to first resolution of delirium may be interpreted as a less rigorous measure, because there is no standard definition for delirium resolution, and delirium is a condition that waxes and wanes on its own.

A post hoc analysis by Devlin et al. was conducted on the above study to compare duration and time to first resolution of 10 delirium symptoms in 29 patients. ${ }^{21}$ Symptoms included agitation, decreased level of consciousness, inattention, disorientation, hallucinations/delusions, hyperactivity, hypoactivity, inappropriate speech or mood, sleep/wake cycle disturbance, and symptom fluctuation. Only symptom fluctuation $(P=0.009)$, time to first resolution of symptom fluctuation $(P=0.004)$, time with inattention (47 vs 78 hours, $P=0.025$ ), and time with symptom fluctuation (47 vs 89 hours; $P=0.04$ ) reached statistical significance with quetiapine compared to placebo. However, quetiapine subjects had a longer time to resolution of agitation ( 3 vs 1 day, $P=0.04$ ) and hyperactivity ( 5 vs 1 day, $P<0.04$ ). The authors attribute these findings to the higher use of as-needed haloperidol in the placebo group ( $1.9 \mathrm{vs} 4.3 \mathrm{mg}$ per day, $P=0.26$ ). These results may also be due to a limitation in the study design, which self-selected for agitation delirium by requiring the use of as-needed haloperidol as inclusion criteria, as symptoms of agitation would be more likely to receive as-needed haloperidol doses. In addition, subjects were allowed to receive 1 to $10 \mathrm{mg}$ of haloperidol up to every 2 hours as needed, but there is no discussion of controlling total daily haloperidol dose in each group in the study. Although 1.9 versus $4.3 \mathrm{mg}$ per day is neither statistically nor likely clinically significant, haloperidol is a treatment for delirium, so the study design would be stronger if quetiapine could be directly compared to an equivalent daily dose of haloperidol or if both groups received the same daily dose of haloperidol. Study results are also limited by the nature of a post hoc analysis, missing documentation for individual delirium symptoms, symptoms of delirium not being well characterized and subjective, and delay in enrollment of subjects.

\section{Noncontrolled Trials}

Six additional trials are included in the table of this review including 5 open label trials and 1 retrospective cohort study. ${ }^{24-29}$ Schwartz and Masand ${ }^{24}$ and Lee et al. $^{29}$ compared the efficacy of quetiapine to 
haloperidol and amisulpride, a second-generation antipsychotic unavailable in the United States, respectively. In a retrospective cohort of 22 general hospital patients, Schwartz and Masand found quetiapine (average dose, $211.4 \mathrm{mg} /$ day) was as efficacious as haloperidol (average dose, $3.4 \mathrm{mg} /$ day) in improving delirium rating scale (DRS) scores by more than $50 \%$ in 10/11 subjects in each group. Lee et al. also found quetiapine (average dose, $113.0 \mathrm{mg} / \mathrm{day}$ ) to be equally efficacious to amisulpride ( $156.4 \mathrm{mg} /$ day) in statistically significantly improving DRS-R-98 scores in 31 neurosurgery, orthopedic surgery, internal medicine, neurology, and rehabilitation medicine patients. Four open-label studies tested the efficacy of flexible doses of quetiapine in a total of 63 general hospital, neurosurgery, orthopedic surgery, and oncology patients. ${ }^{25-28}$ Pae et al., ${ }^{25}$ Maneeton et al., ${ }^{26}$ and Kim et al. ${ }^{27}$ found that quetiapine statistically significantly reduced DRS-R-98, Clinical Global Impression ScaleSeverity, DRS, MMSE, and Clock Drawing Test scores from baseline. Sasaki et al. ${ }^{28}$ found all 12 general hospital patients in their study reached remission with an average daily quetiapine dose of $44.9 \mathrm{mg} / \mathrm{day}$. Although these studies have a limited level of evidence due to their small sample sizes, study designs, and heterogeneous subjects, they do still suggest that quetiapine may effectively treat delirium in various patient populations.

\section{DISCUSSION}

Although the role of quetiapine for the treatment of delirium continues to develop, the studies evaluated here suggest that quetiapine may be effective and safe for this usage. Resolution of delirium from baseline was shown in all studies. Quetiapine resolved symptoms of delirium more quickly than placebo and had equal efficacy to other antipsychotics, haloperidol and amisulpride. Quetiapine may be most useful for patients with symptom fluctuation as opposed to agitation and hyperactivity or in those patients who may not tolerate haloperidol well. Both randomized control and open-label trials found a low incidence of adverse effects with quetiapine. There were fewer incidences of QT prolongation and extrapyramidal symptoms, but higher a rate of somnolence with quetiapine compared to other antipsychotics in these trials. However, none of these differences in adverse effects reached statistical significance.

Drawing definitive conclusions about the efficacy of quetiapine in the treatment of delirium is difficult due to multiple study limitations. First, the body of literature is small, with only 2 randomized controlled trials, both of which had limitations. Tahir et al. ${ }^{19}$ was underpowered and found no statistically significant difference in the primary end point, DRS-R-98 total mean score. Devlin et al. ${ }^{20}$ tested the primary end point of time to first resolution of delirium, which may be interpreted as a less rigorous measure because there is no standard definition for delirium resolution. Furthermore, both randomized controlled and observational trials tested the efficacy of quetiapine using different tests, making comparison between these trials difficult. All studies included in this review were carried out in small patient populations, with the largest trial having 42 subjects, and had highly restrictive exclusion criteria limiting the generalizability of the study population to the general hospital population. Although most of the patients included in these studies are general hospital populations, Devlin et al. was performed in critically ill patients, which creates the additional limitation of having heterogeneous study populations. Last, superiority of any 1 antipsychotic is not possible given that none of these studies have done a head-to-head comparison of quetiapine with another atypical antipsychotic.

Given the comparable efficacy and safety of antipsychotics, cost is a relevant factor in treatment decisions. Haloperidol is supplied as either a suspension for injection or a tablet. One vial of $5 \mathrm{mg} / \mathrm{mL}$ haloperidol is $\$ 8.32$. Haloperidol $5 \mathrm{mg}$ tablets are approximately $\$ 0.29$ each, whereas $25 \mathrm{mg}$ tablets of quetiapine are approximately $\$ 3.53$ each. However, these prices are for consumers and will vary depending on institutional contracts. ${ }^{42}$

\section{CONCLUSION}

Quetiapine appears to be an effective and safe agent for the treatment of delirium in both general medicine and ICU patients. Superiority of quetiapine over other antipsychotics for hospital-associated delirium has not been shown due to limitations in quality and quantity of data. Large, randomized, double-blind, active control studies with longer study durations are needed to elucidate the efficacy and niche of quetiapine in the treatment of delirium.

Disclosure: Nothing to report.

\section{References}

1. American Psychiatric Association. Diagnostic and Statistical Manual of Mental Disorders. 4th ed. Washington, DC: APA; 1994.

2. American Psychiatric Association. Practice guideline for the treatment of patients with delirium. Am J Psychiatry. 1999;156:1-20.

3. Hipp DM, Ely EW. Pharmacological and nonpharmacological management of delirium in critically ill patients. Neurotherapeutics. 2012:9:158-175.

4. Stiefel F, Holland J. Delirium in cancer patients. Int Psychogeriatr. 1991;3:333-336.

5. Perry S. Organic mental disorders caused by HIV: update on early-diagnosis and treatment. Am J Psychiatry. 1990;147:696-710.

6. Tune LE. Post-operative delirium. Int Psychogeriatr. 1991;3:325-332.

7. Massie MJ, Holland J, Glass E. Delirium in terminally ill cancer patients. Am J Psychiatry. 1983;140:1048-1050.

8. Seitz DP, Sudeep SG, van Zyl LT. Antipsychotics in the treatment of delirium: a systematic review. J Clin Psychiatry. 2007;68:11-21.

9. Inouye S, Horowitz R, Tinetti M, et al. Acute confusional states in the hospitalized elderly: incidence, risk factors and complications [abstract]. Clin Res. 1989;37:524A.

10. Cole MG, Primeau FJ. Prognosis of delirium in elderly hospital patients. CMAJ. 1993;149:41-46.

11. Koizumi J, Shiraishi H, Suzuki T. Duration of delirium shortened by the correction of electrolyte imbalance. Jpn I Psychiatry Neurol. 1988;42:81-88. 
12. Jacobi J, Fraser GL, Coursin DB, et al. Clinical practice guidelines for the sustained use of sedatives and analgesics in the critically ill adult. Crit Care Med. 2002;30:119-141.

13. Thomason JW, Shintani A, Peterson JF, Pun BT, Jackson JC, Ely EW. Intensive care unit delirium is an independent predictor of longer hospital stay: a prospective analysis of 261 non-ventilated patients. Crit Care. 2005;9:R375-R381.

14. Milbrandt EB, Deppen S, Harrison PL, et al. Costs associated with delirium in mechanically ventilated patients. Crit Care Med. 2004;32:955-962.

15. Cook IA: Guideline Watch: Practice Guideline for the Treatment of Patients with delirium. Arlington, VA: American Psychiatric Association, 2004. Available at: http://www.psych.org/psych_pract/treatg/pg/ prac_guide.cfm. Accessed March 5, 2012.

16. Han C, Kim YK. A double-blind trial of risperdone and haloperidol for the treatment of delirium. Psychosomatics. 2004;45:297301.

17. Skrobik YK, Bergeron N, Dumont M, Gottfried SB. Olanzapine vs haloperidol: treating delirium in a critical care setting. Intensive Care Med. 2004;30:444-449.

18. Girard TD, Pandharipande PP, Carson SS, et al. Feasibility, efficacy, and safety of antipsychotics for intensive care unit delirium: the MIND randomized, placebo-controlled trial. Crit Care Med. 2010;38:428-437.

19. Tahir TA, Eeles E, Karapareddy V, et al. A randomized controlled trial of quetiapine versus placebo in the treatment of delirium. Psychosom Res. 2010;69:485-490.

20. Devlin JW, Roberts RJ, Fong JJ, et al. Efficacy and safety of quetiapine in critically ill patients with delirium: a prospective, multicenter, randomized, double-blind, placebo-controlled pilot study. Crit Care Med. 2010;38:419-427.

21. Devlin JW, Skrobik Y, Riker RR, et al. Impact of quetiapine on resolution of individual delirium symptoms in critically ill patients with delirium: a post-hoc analysis of a double-blind, randomized, placebocontrolled study. Critical Care. 2011;15:R215.

22. Sipahimalani A, Masand PS. Olanzapine in the treatment of delirium. Psychosomatics. 1998;39:422-430.

23. Grover S, Kumar V, Chakrabarti S. Comparative efficacy study of haloperidol, olanzapine and risperidone in delirium. J Psychosom Res. 2011;71:277-281.

24. Schwartz TL, Masand P. Treatment of delirium with quetiapine. J Clin Psychiatry. 2000;2:10-12.

25. Pae CU, Lee SJ, Lee CU, Lee C, Paik IH. A pilot trial of quetiapine for the treatment of patients with delirium. Hum Psychopharmacol Clin Exp. 2004;19:125-127.

26. Maneeton B, Maneeton N, Srisurapanont M. An open-label study of quetiapine for delirium. J Med Assoc Thai. 2007;10:2158-2163.
27. Kim KY, Bader GM, Kotlyar V, Gropper, D. Treatment of delirium in older adults with quetiapine. J Geriatr Psychiatry Neurol. 2003;16:29-31.

28. Sasaki Y, Matsuyama T, Inoue S, et al. A prospective, open-label, flexible-dose study of quetiapine in the treatment of delirium. J Clin Psychiatry. 2003;64:1316-1321.

29. Lee KU, Won WY, Lee HK, et al. Amisulpride versus quetiapine for the treatment of delirium: a randomized, open prospective study. Int Clin Psychopharmacol. 2005;20:311-314.

30. Campbell N, Boustani MA, Ayub A, et al. Pharmacological management of delirium in hospitalized adults-a systematic evidence review. $J$ Gen Intern Med. 2009;24:848-853.

31. Lacasse H, Perreault MM, Williamson DR. Systematic review of antipsychotics for the treatment of hospital-associated delirium in medically or surgically ill patients. Ann Pharmacother. 2006;40:1966-1973.

32. Rea RS, Battistone S, Fong JJ, Devlin JW. Atypical antipsychotics versus haloperidol for treatment of delirium in acutely ill patients. Pharmacotherapy. 2007;27:588-594.

33. Cole MG, Primeau FJ, Elie LM. Delirium: prevention, treatment, and outcome studies. J Geriatr Psychiatry Neurol. 1998;11:126-137.

34. Saller CF, Salama AI. Seroquel: biochemical profile of a potential atypical antipsychotic. Psychopharmacology. 1993;112:285-292.

35. Seroquel [package insert]. Wilmington, DE: AstraZeneca Pharmaceuticals LP; December 2011.

36. Harrigan EP, Miceli JJ, Anziano R, et al. A randomized evaluation of the effects of six antipsychotic agents on QTc, in the absence and presence of metabolic inhibition. J Clin Psychopharmacol. 2004;24:6269.

37. Kales HC, Valenstein M, Kim HM, et al. Mortality risk in patients with dementia treated with antipsychotics versus other psychiatric medications. Am J Psychiatry. 2007;164:1568-1576.

38. Gill S, Bronskill SE, Normand SL, et al. Antipsychotic drug use and mortality in older adults with dementia. Ann Intern Med. 2007;146:775-786.

39. Wang PS, Schneeweis S, Avorn J, et al. Risk of death in elderly users of conventional vs. atypical antipsychotic medications. N Engl J Med. 2005;353:2335-2341.

40. Ray WA, Chung CP, Murray KT, Hall K, Stein CM. Atypical antipsychotic drugs and the risk of sudden cardiac death. N Engl J Med. 2009;360:225-235

41. Elie M, Boss K, Cole MG, McCusker J, Belzile E, Ciampi A. A retrospective, exploratory, secondary analysis of the association between antipsychotic use and mortality in elderly patients with delirium. Int Psychogeriatr. 2009;21(3):588-592.

42. Walgreens Co. Price your drugs Available at: http://www. walgreens.com/pharmacy/psc/drugpricing/psc_drug_pricing.jsp. Accessed December 17, 2012. 